\title{
Valdivia Capital Cultural Americana 2016. Una mirada desde la economía, su realidad y proyecciones
}

\author{
Paulina N. Retamal Yermani \\ Facultad de Filosofía y Letras \\ Universidad de Buenos Aires \\ pryermani@gmail.com
}

Recibido: $12 / 03 / 2015$

Modificado: $25 / 05 / 2015$

Aceptado: 26/05/2015

\section{Resumen}

Este artículo aborda la relación entre ciudad y economía de la cultura usando como referencia la austral ciudad de Valdivia, situada en el sur de Chile. A propósito de este nexo, se busca abrir la discusión sobre su importancia, la incidencia y los posibles escenarios en el contexto de la Declaratoria Ciudad Americana 2016, organizada por el International Bureau of Cultural Capitals. Esta declaratoria escoge una ciudad de América por año para ser representante de la vida cultural, con el objetivo de potenciar las actividades, las industrias creativas y aumentar el turismo cultural en la zona. Elaborando discusiones teóricas con literatura existente al respecto, se persigue vislumbrar la incidencia de dicha declaratoria en la ciudad de Valdivia usando como referencia la experiencia de ciudades análogas anteriormente declaradas.

Palabras clave: ciudad, Valdivia, economía, cultura.

Title: Valdivia, American Capital of Culture 2016. A view from the perspective of its economy, reality and projections

\section{Abstract}

This article discusses the relationship between city and culture economy using as a reference the city of Valdivia located in southern Chile. With regard to this relationship, we discuss the importance, incidence and possible scenarios in the context of the Declaratoria Ciudad Americana 2016 , organized by the International Bureau of Cultural Capitals. This is an initiative which selects one city in America per year as representative of cultural life, with the aim of enhancing creative activities and industries and of increasing cultural tourism in the area. Elaborating theoretical discussions based on the existing literature, this is an attempt to discern the effect of this initiative on the city of Valdivia with reference to the relevant experience of the cities which had been previously selected.

Keywords: city, Valdivia, economy, culture.

\section{Índice}

1. Ciudad creativa: la economía cultural de los espacios

2. Ciudad Americana de la Cultura

3. Una mirada al futuro de la economía creativa en Valdivia 


\section{Ciudad creativa: la economía cultural de los espacios}

En las ciudades, la relación con la economía no es reciente. Este vínculo entre las dinámicas urbanas y las económicas se debe, históricamente, al hecho mismo de que la ciudad se concibe como el espacio dedicado a la economía por excelencia. Se trata de la forma en que el sistema de libre mercado encuentra en ella su nicho de producción y de re-producción de sí mismo. Así, tal como afirma Gómez (2004: 37), "las ciudades son entonces expresión del orden económico y social neoliberal que entiende el desarrollo en términos de producción, distribución y consumo". En efecto, la idea primaria de ciudad está íntimamente asociada a la de economía, sobre todo porque representa el soporte de las operaciones económicas. A su vez, cuando pensamos la triada economía/ciudad/cultura ${ }^{1}$, se potencian un sinfín de actividades y movimientos que confluyen en distintos puntos y con distintos objetivos.

[...] en este sector [cultural] es fundamental la confluencia de trabajos creativos, de fabricación y de organización sobre materiales simbólicos que vehiculizan significaciones sociales, generando, comunicando y activando en esos mismos procesos ciertos sentidos en detrimento de otros que quedan no visibilizados u oscurecidos. (Bayardo 2013: 17)

De este modo, la ciudad se transforma en el centro neurálgico, de producción mercantil simbólica en palabras de Getino. Finalmente es donde se generan los imaginarios y materiales simbólicos que posteriormente circulan por la ciudad, como también aquéllos que se logran exportar fuera de ella. Esta manera de generación ligada a la producción de símbolos, puede vincularse con el concepto de economía creativa, que si bien no es una forma primaria de establecer lazos entre cultura y economía, sí se considera el concepto más avalado. La economía creativa, además, está fuertemente relacionada con la explotación de la creatividad y el talento individual, así como también la generación de propiedad intelectual y copyright, cuyo mecanismo de operación es el autoempleo, el emprendimiento por medio de la inclusión de pequeñas y medianas empresas. Dicha economía creativa trasmuta la concepción en el espacio urbano respecto a su acervo cultural, así podríamos dar una primera definición de economía creativa siguiendo a Yúdice, que la define como:

\footnotetext{
${ }^{1}$ Entendemos por área cultural: literatura, artes y patrimonio; artesanías, folklore y expresiones populares como fiestas y celebraciones; industrias culturales y medios. Más recientemente, puede incluir moda, diseño, gastronomía ("artes culinarias"), organizaciones sociales territoriales e indentitarias, turismo cultural (Bayardo 2013: 18).
} 
[...] actividades como el turismo cultural y el desarrollo de las artes también contribuyen a la transformación de las ciudades posindustriales, la que, junto al trabajo de innovación, desarrolla el tejido social de bares, restaurantes, encuentros en la calle, etc., que da vida a este lugar. (Yúdice 2012: 34)

Para ofrecer una ejemplificación más acabada de los sectores que componen la economía creativa, citaremos a David Throsby con la teoría del modelo de círculos concéntricos. Con esta teoría, el autor pone el "valor cultural de los bienes" como punto distintivo, en tal sentido, si un valor es más potenciado, más crece el argumento para incluir a la economía que lo produce. Ese valor se produce en el núcleo de este diagrama y se va difundiendo a los círculos más externos del esquema (figura 1 ).

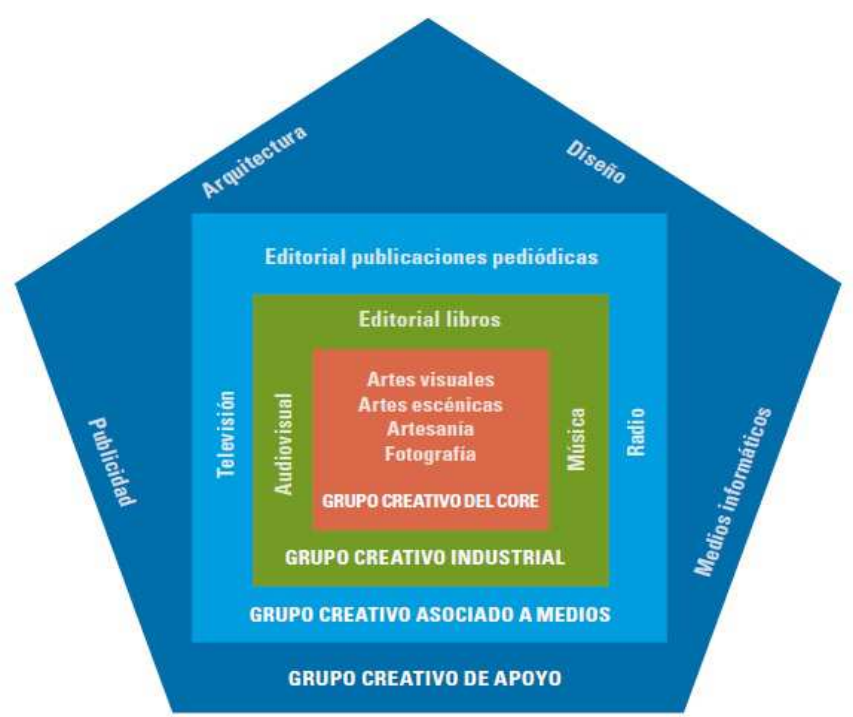

Figura 1. Agrupación de sectores creativos según el modelo de círculos concéntricos de D. Throsby. Fuente: Consejo Nacional de la Cultura y las Artes, Santiago, Chile.

Desde el urbanismo y la cultura, un autor español avala la teoría de Throsby de la conformación esférica de la cultura, explicando que en la ciudad comprende como tal "[...] un entramado de círculos concéntricos de significados, prácticas e influencias que van imbricándose, sustituyéndose unas a otras y transformándose según evoluciona el modelo, pero sin dejar atrás nunca de ser a su desarrollo e imprescindibles para su análisis" (Balibrea 2011).

De este modo, se potencia la calidad de vida y de circulación en la ciudad, situación que la vuelve permeable a atraer y retener a los agentes emprendedores e innovadores, indispensables para la nueva economía creativa. En consecuencia, las urbes y, por lo tanto, Estados, gobiernos y organizaciones, han considerado a la cultura en 
conjunto con la economía como un modo rentable y viable de manejar las ciudades.

Del sustento fáctico de la economía creativa devienen las industrias creativas, que vienen a potenciar las relaciones en la ciudad por medio de la cadena de creación, producción, distribución y comercialización de bienes y servicios simbólicos. Según la UNESCO, las industrias creativas se caracterizan por combinar creación, producción y comercialización de contenidos que son intangibles y culturales en su naturaleza. Como explica Getino (2007: 2), las industrias culturales no sólo son un aporte a nivel económico allá donde se las sitúa, sino que potencian el crecimiento social del lugar en donde se emplazan, por tanto, tienen una "función fundamental en la creación de los imaginarios individuales y de las identidades colectivas, y constituyen uno de los sectores principales de expresión y diálogo entre las culturas".

En síntesis, en las ciudades creativas convergen estos puntos revisados anteriormente -a saber, economía e industrias creativas-, transformándose la ciudad en el soporte para que esta serie de actividades se lleven a cabo siempre con miras a la economía y el progreso. Esto se concreta en una unión entre las prácticas originarias y las ideas que se mantengan vigentes sobre comunidad y desarrollo económico; por lo tanto, con enfrentamos a una maquinaria imaginaria en donde su "funcionamiento observamos en las ciudades globales que concentran oficinas de mando y control para las corporaciones transnacionales, y una masa crítica concomitante de servicios complementarios y avanzados al productor" (Yúdice 2012: 35).

\section{Ciudad Americana de la Cultura}

Valdivia es una ciudad lacustre ubicada a ochocientos kilómetros al Sur de Santiago de Chile, capital de la Región de los Ríos (XIV región) que se destaca por su multiplicidad de manifestaciones artísticas y culturales, así como por la riqueza natural que la caracteriza. En ella confluyen culturas autóctonas (cultura tradicional mapuche); cultura española y mestiza, producto de los siglos de estadía peninsular en la zona; cultura alemana, cuya origen se halla en el siglo XIX, como parte de un programa implementado por el gobierno de Chile para paliar la crisis económica de la zona, cuyo objetivo fue atraer inmigrantes alemanes con el fin de generar una economía manufacturera más robusta; y actualmente, variadas culturas provenientes del resto del país (sobre todo jóvenes profesionales de la capital que migran al Sur en busca de posibilidades laborales y académicas). Este fenómeno de capas da un excelso atractivo cultural y turístico, característica que las autoridades de la zona, así como organizaciones sociales han detectado, con el fin de potenciar la oferta turística, artística y cultural de la región. 
En junio de 2014 la ciudad de Valdivia fue declarada como "Capital Americana de la Cultura" por un organismo español llamado Bureau Internacional de Capitales Culturales (IBOCC, según las siglas en inglés), encabezado por su presidente Xavier Tudela, junto con la Ilustre Municipalidad de Valdivia. Capital Cultural Americana de la Cultura fue fundada en España a fines de los años noventa por su actual presidente con el objetivo de promover la "integración interamericana desde el ámbito cultural, contribuir a un mejor conocimiento entre los pueblos del continente americano, respetando su diversidad nacional y regional, poniendo de relieve al mismo tiempo el patrimonio cultural común" (International Bureau of Cultural Capitals 2014), así como también vincular internacionalmente a ciudades americanas con Europa.

Nuestra problemática se enfoca sobre las posibilidades reales e hipotéticas a corto, medio y largo plazo que ofrecería esta declaratoria a la ciudad, así como considerar si los agentes que intervienen en las decisiones culturales de la ciudad poseen el conocimiento necesario para fomentar la imagen de la ciudad en el mundo. Una primera mirada se enfrentaría a una declaratoria más bien estéril por lo deficiente de su alcance y respaldo. Sin embargo, esta situación podría ser revertida si los encargados de gestionar cultural y turísticamente la ciudad aprovecharan dicha instancia como catalizadora de un proceso en el cual vienen interviniendo hace muchos años.

En términos legales, esta declaración no posee ni respaldo ni validez de organizaciones o instituciones oficiales (como la Unesco o Mercosur, entre otras), además de no aportar económicamente a la ciudad designada que potencie el desarrollo cultural. La modalidad consiste en la postulación de distintas ciudades, las cuales deben pagar una adhesión por participar. Al respecto, no se tiene conocimiento ni de quién es el jurado, ni de cómo es el proceso de selección, como tampoco se conocen cuáles son las ciudades que se postulan cada año. Sin embargo, pese al ingrávido peso que esta declaratoria posee, para las ciudades este hecho viene a potenciar sus posibilidades económicas, sobre todo en lo que respecta a la materia cultural. Enseguida salta la duda sobre la legitimidad de esta declaratoria, ya que se trata de un organismo europeo el que decide qué ciudad y por qué debe ser considerada "representante cultural" de la región. En Cultura y desarrollo. Lo que aporta la cultura a la economía, Tolila (2007: 90) apunta:

la atención de los tomadores de decisiones y de sus representantes se orientó en dos direcciones clásicas en el marco de las democracias: por una parte, el debate público interno para la asignación de los recursos, su valor y sus significados, y por otra la competencia externa con los demás Estados en materia de mercados 
y de comercio $[\ldots]$, procesos y los debates que animan hoy todas las reflexiones sobre la globalización y la diversidad de las expresiones culturales.

La mirada eurocentrista en términos de cultura se sigue potenciando, aun cuando las ciudades americanas están tomando en sus manos estos conflictos -y sus resoluciones-, sin embargo, el antecedente del hemisferio norte todavía sigue marcando las directrices de los resultados económicos. Posiblemente, para estas ciudades resulta beneficioso, incluso pagando esta cuota de postulación, el hecho de enlistarse en este concurso, como una jugada estratégica por parte de las autoridades, haciendo que público europeo, norteamericano y asiático elija como destinos turísticos y culturales ciudades de Latinoamérica. Finalmente, para las mismas ciudades, pagar por estar presentes en este concurso $y$, posteriormente, ser escogidos por la comisión supone una inversión, puesto que una vez obtenida la declaratoria se garantizan una amplia difusión por los canales oficiales; lo que atraería a una serie de turistas con alto poder adquisitivo, que visitarían estas ciudades e invertirían sus divisas en cultura, engrosando las estadísticas cuantitativas de la economía creativa.

El documento del IBOCC hace hincapié en importantes temas de economía, cultura y ciudad, detectando el potencial que posee la ciudad de Valdivia en temas de economía:

Valdivia se reconvierte como una ciudad universitaria y turística, que poco a poco evoluciona hacia la meta de convertirse en un polo cultural y científico. Sin duda este nombramiento desencadenará su potencial cultural, atraerá las miradas del continente y consolidará los esfuerzos institucionales propios tal y como está ocurriendo con Colima 2014. (International Bureau of Cultural Capitals 2014)

Estas palabras confirman que Valdivia ha realizado un minucioso camino en pos de aumentar, generar y difundir las creaciones artísticas, así como los adelantos en materia de ciencias fácticas, con la intención de potenciar la transformación de la ciudad en un polo de desarrollo económico, usando como guía la noción de ciudades creativas, en donde se genere una urdimbre de actividades, servicios, productos y relaciones económicas ligadas a la cultura.

Cabe preguntarse al respecto, ¿qué consecuencias económicas, vistas desde la cultura, podría acarrear esta declaración para la ciudad?, ¿qué incidencias culturales, favorables y perjudiciales se proyectan?, ¿qué imagen de arte, cultura y turismo se generará sobre Valdivia en Chile y en el extranjero?, ¿qué se espera de los valdivianos, de los organismos públicos y privados, y de la sociedad chilena en general? Responder estas preguntas encaminaría las posibles consecuencias en economía cultural de esta región. 


\section{Una mirada al futuro de la economía creativa en Valdivia}

Sin duda, las instituciones de la zona, tanto privadas como públicas, han recibido positivamente la noticia de esta declaración. La Municipalidad de Valdivia, encabezada por el alcalde, en conjunto con la ciudadanía -que incluye agrupaciones y el ámbito universitario-, está preparando una completa agenda de actividades para el año 2016 que permitirá visualizar claramente la cultura como un elemento de inclusión ciudadana, transformación social y desarrollo económico. Persiguen consolidar una imagen de ciudad económica capaz de potenciar sus recursos actualmente existentes, atraer agentes de innovación y resaltar el nexo entre las empresas e industrias creativas, en conjunto con el recurso humano pensante. Este talento humano es el que debe captarse: "Para atraer a ese talento, las ciudades deben ofrecer una alta calidad de vida, lo cual significa que estas son también generadoras mayores de capital y valor culturales" (Yúdice 2012: 35). Durante el año 2014, la comisión investigadora del Consejo Nacional de la Cultura y las Artes (CNCA) realizó el primer estudio titulado Mapeo de las Industrias Creativas en Chile: caracterización y dimensionamiento, que pretendía delimitar qué se entendería como "industrias creativas" en el país bajo la imagen del CNCA, así como también obtener una serie de diagnósticos validados por los agentes que conforman dichas industrias. El estudio explica que se han detectado ciertas características particulares de las industrias creativas que desentonan respecto a otro tipo de industrias económicas más tradicionales, por ejemplo, aclaran que es un sector que necesariamente nace y se nutre del territorio que lo sustenta, destacando la presencia de su fuerza laboral: "donde quienes trabajan lo hacen muchas veces por vocación -por lo que las remuneraciones y ventas no necesariamente reflejan el beneficio real que las actividades creativas generan-, un sector que requiere de estímulos y educación previa para la generación de audiencias, y en el que las externalidades producidas son siempre positivas" (Aspillaga Fariña 2014). El estudio confirma que tanto el lugar de emplazamiento como su talento humano juegan un papel primordial en el futuro de este tipo de industrias. El hecho de que los agentes culturales deban postergar sus salarios en virtud de generar espacios o instancias culturales más propicias para las ciudades, confirma el fenómeno de que para esta declaratoria fue necesario que la ciudad pagara su inscripción al concurso. Esto imposibilita que sectores con menor -o incluso nula- capacidad económica que el sector cultural de una ciudad puedan acceder a esferas globales de instancias de legitimación simbólica. La viabilidad de este proceso se explica porque el agente que postula a la ciudad como candidata es la Municipalidad de Valdivia, que es representante local del Estadio Nacional, teniendo el respaldo de la jurisprudencia 
del país. Por otro lado, la buena noticia es visible por el hecho de que las industrias creativas están siendo consideradas como parte importante del desarrollo económico del país, situación que facilitaría el acceso de éstas a las políticas públicas. La economía de la cultura está usándose sobre todo con fines balsámicos, cuya vía de escape está relacionada con la acción de salvación más que con la operación coyuntural.

En este sentido, en la última década, se evidencia un aumento de los fondos adquiridos por industrias creativas que se entregan por medio de concurso público a nivel nacional (Fondo Nacional para la Cultura y las Artes, Fondart). Esto manifiesta, en materia económica, la importancia de las industrias culturales en proyecciones monetarias para el país. Es decir, esto significa para los fondos nacionales una inversión, al destinar recursos públicos a estas industrias para que luego contribuyan al desarrollo económico como contraprestación.

\begin{tabular}{|c|r|c|}
\hline Año & Montos lineas industrias & Porcentaje respecto al total \\
\hline 2004 & 579.684 .660 & $9 \%$ \\
\hline 2005 & 1.673 .455 .817 & $25 \%$ \\
\hline 2006 & 2.058 .581 .592 & $26 \%$ \\
\hline 2007 & 4.011 .391 .386 & $39 \%$ \\
\hline 2008 & 3.336 .326 .749 & $32 \%$ \\
\hline 2009 & 4.267 .276 .978 & $33 \%$ \\
\hline 2010 & 3.074 .032 .444 & $25 \%$ \\
\hline
\end{tabular}

Figura 2. Montos en pesos chilenos destinados a las Industrias Creativas en Chile. (Referencias monetarias: el monto más bajo corresponde a 970.000 dólares mientras que el más alto a 5.000.000 dólares). Fuente: Consejo Nacional de la Cultura y las Artes, Santiago, Chile.

En efecto, para lograr este nivel de vida deseado -en donde industria y talento creativo convivan-, se espera que los habitantes de Valdivia experimenten una mayor valoración de sus tesoros culturales y una toma de conciencia de lo regional frente a lo universal. Se hace latente entonces la cultura como vehículo de la economía, pasa a ser un sector más del macropanorama de la economía en la medida en que las ciudades tienden cada vez más a la oferta de servicios y experiencias como nuevo producto de consumo. Por ello la tríada cultura/creatividad/conocimiento "va siempre de la mano, potenciando la explotación de un tipo de capital humano cualificado del que se espera que cree, ya sea arte y cultura, ya sea ciencia o tecnología en universidades, laboratorios, etc." (Balibrea 2011). Ejemplo de esto es la Universidad de Valdivia, espacio por antonomasia que contribuye a la generación de material artístico como científico con el propósito de demostrar que ambas actividades 
intelectuales enriquecen, engrandecen y potencian la imagen simbólica de una ciudad.

Desde esta perspectiva social, el impacto que se espera a nivel humano es que los ciudadanos se sientan partícipes en la construcción de un nuevo significado del ser valdiviano y el ser latinoamericano por medio de la cultura. En efecto, lo que se estima es que las personas adapten su comportamiento social en virtud de potenciar la ciudad, esto favorecerá

[...] evidentemente a la clase profesional-gerencial por cuanto saca provecho de la retórica de la inclusión multicultural. Los grupos subordinados y minoritarios ocupan un lugar en este esquema en calidad de obreros no calificados que aportan servicios y en calidad de proveedores de "vida" étnica y de otras experiencias culturales que "representan el nuevo estadio del desarrollo capitalista". (Yúdice 2012: 35)

La culturización de la ciudad se trasformará en una forma de manejo de la población, principalmente las zonas populares, ya que potencian la vida del lugar mientras nutren innovaciones de los creadores. En paralelo, además, la economía de la cultura pretende jugar un importante papel en lo que respecta a la sociedad, pues contribuye en "los procesos de integración nacional y regional, además de lo que puede significar para la identidad y el autoreconocimiento de los individuos y las sociedades" (Getino 2007: 4).

Como consecuencia, se espera lograr nuevos espacios de confluencia cultural, tal como pasó en Barranquilla, Ciudad Americana de la Cultura 2013, que la alcaldesa aseguró "haber cerrado con un balance positivo para la cultura en nuestra ciudad". A nivel ciudad se proyecta la construcción de nuevos espacios urbanos que respondan a las necesidades culturales, tales como parques, plazas y galerías, que convoquen a la ciudadanía. La cultura se expande a nuevos espacios, ahora abarca espacios públicos y privados, como establecimientos educacionales, así como también plazas y espacios verdes, hoteles, oficinas comerciales, centros de recreación, que han integrado a sus arcas a instituciones artísticas, una serie de "organismos de bienestar social que sirven, todos ellos, para resaltar los aspectos utilitarios de las artes en la sociedad contemporánea" (Larson 1997: 127-128).

Otra de las consecuencias que impactará inmediatamente, es la garantía que ofrece IBOCC como modo de difusión de la ciudad de Valdivia vía canales satelitales y por plataformas digitales. Se desea potenciar internacionalmente, a través de la señal Antena 3 TV de Madrid. Al respecto, Mar Martínez-Raposo, Gerente del Canal Internacional, ha declarado que "desde hace trece años Antena 3 
Internacional colabora como canal oficial en la promoción y difusión de la capitalidad cultural, tanto en Iberoamérica como en el ámbito hispano de los Estados Unidos de América y en Europa". Así mismo, será transmitido ampliamente por la cadena Discovery Network. Ambas cadenas, de alcance internacional, apuntan a un tipo de audiencia específica, la cual, además, posee sus prioridades en términos de consumo cultural y destinos turísticos. Si se desea potenciar la imagen de Valdivia en los mass media, pasando del anonimato local al reconocimiento internacional, los desafíos en materia de presupuesto, calendarización y proyectos en economía cultural y también creativa, debieran apuntar a una estricta planificación que entregue ganancias económicas como también simbólicas que enriquezcan el desarrollo de la ciudad. La cultura es el negocio de las ciudades, convirtiéndose éstas en espacios donde se reformula la idea de lo nacional y de cultura común. Las ciudades modifican sus territorios originales reformulando sus dinámicas para generar un nuevo espacio económico determinado principalmente por la aglomeración de industrias creativas que otorgarán a las ciudades una identidad recién fundada ${ }^{2}$. Se crean mecanismos que indican la concepción nacional: son nuevas formas de organizar culturalmente un territorio. Uno de los aspectos que modifica este ordenamiento citadino en virtud de funcionalismos culturales -ciudad del arte, de la música, la gastronomía o el diseño, etc.- es el turismo cultural.

Como evidencia Tolila (2007), la relación del turismo con la economía de la cultura "suele ser la primera idea de análisis económico de la cultura. La argumentación en términos de impacto es interesante pero limitada al ser una lógica cuantitativa lineal, cuya fuerza disminuye en cuanto se aleja de los impactos directamente observables y cuantificables". Evidentemente uno de los impactos con mayor expectativa es la proyección en materia de turismo cultural. El binomio cultura y economía se ha resuelto en la mayoría de los casos bajo la imagen del turismo cultural. Esta relación es la que finalmente justifica los estudios con indicadores económicos dentro de las investigaciones en economía de la cultura. En efecto, instituciones públicas, privadas, así como iniciativas cívicas persiguen está vía como modo de obtención de divisas por medio de esta área, "sea para estimular el crecimiento económico mediante proyectos de desarrollo cultural urbano y la concomitante proliferación de museos cuyo fin es el turismo cultural" (Yúdice 2012: 24). La ciudad de Valdivia ya se reconoce por su importante legado patrimonial, tanto histórico como cultural y natural. Sin lugar a dudas, este reconocimiento permitirá que la ciudad logre destacar aún más sus

2 Por ejemplo, Londres, capital de las finanzas; Ámsterdam, capital cultural y de ocio, Barcelona, capital del conocimiento, entre otras. 
herencias en este aspecto, potenciando el trabajo que se realiza desde el Servicio Nacional de Turismo bajo el alero de la Política Regional de Turismo aprobada por el Consejo Regional de Los Ríos. Se evidencia una clara intención de incorporar el turismo cultural y patrimonial como eje de trabajo.

En economía creativa, "sus principales actores son las clases creativas, y su lugar por excelencia son ciudades, regiones y clusters creativos, especialmente en 'países en desarrollo', pues se concibe que estos no exploran suficientemente sus recursos, y cuentan con ingentes 'reservas de talento' disponible" (Bayardo 2013: 21). Los futuros clusters que pretenden generarse a propósito de esta declaratoria son el punto más fuerte de esperanza puesta en la ciudad en análisis. Aún se está buscando la marca de Valdivia, ya que en la ciudad se desarrollan un sinfín de actividades culturales. Incluso la variedad de oferta cultural en una zona austral del mundo puede transformarse en el sello de la ciudad. Chile, como país en desarrollo, busca dentro de sus posibilidades la realidad de explotar sus reservas de talentos que se están gestando.

La declaratoria de Ciudad Americana de la Cultura se comporta como un vigoroso empujón para potenciar las industrias creativas de la zona en pos de un desarrollo económico más robusto para la región. Luego del quiebre de las empresas salmoneras a principio de la década del 2000, y el cierre de una serie de industrias de celulosas acusadas de altos niveles de contaminación ambiental ${ }^{3}$, sumado a la reciente división territorial que posicionó a Valdivia como capital regional de Los Ríos, tanto la dimensión estatal como las iniciativas particulares debieron buscar nuevas fuentes de ingresos que se mantuvieran de forma constante, supliendo el vacío de los años anteriores. Las industrias culturales vinieron a paliar una serie de conflictos económicos y sociales que estaban amenazando la prosperidad de la ciudad.

\footnotetext{
${ }^{3}$ Hacia el año 2004, la celulosa Arauco, son sede en Valdivia, fue puesta bajo la ley por contaminar el Santuario de la Naturaleza Carlos Anwandter, del Río Cruces, en donde la población de cisnes de cuello negro, especie endógena del sur de Chile, se vio disminuida drásticamente. Esto condenó a la celulosa a nivel legal, pero también se deterioró su imagen ante la ciudadanía. Ese proceso legal llevado a cabo por la fiscalía local junto a científicos de la Universidad Austral de Chile, tuvo como consecuencia en primera instancia el cierre de la celulosa, y posteriormente, una caída de la producción en este ámbito, siendo desplazada como fuente de riqueza y empleo de la zona.
} 
Constituyen una forma de conocimiento que se traduce en empleos y abundancia, consolida la creatividad (su "materia prima") para fomentar la innovación en los procesos de producción y comercialización. Al mismo tiempo, son centrales en la promoción y el mantenimiento de la diversidad cultural. Así como para el aseguramiento del acceso democrático a la cultura. (Piedras 2004: 29)

El desafío está en manos del Estado y en las iniciativas particulares y ciudadanas para que la experiencia de esta declaratoria se transforme en una oportunidad para la ciudad, y no en cambio en una amenaza que suponga pérdidas, no sólo económicas, sino también de prácticas humanas, incluso de patrimonios muchas veces irrecuperables. Las consecuencias por tanto de esta declaratoria pueden variar, y la responsabilidad de sus resultados recaerá principalmente en dos agentes: el Estado chileno y sus representantes regionales, quienes deben proyectar el qué, el por qué y el cómo de la serie de acciones y medidas que la región desea tomar en este punto, teniendo en cuenta las causas y consecuencias a nivel de industrias y economía creativa, poniendo énfasis en la potencialidad de las mismas; y por otro lado, encontramos la labor de los gestores culturales, tanto a nivel privado como público, quienes debieran detectar a tiempo esta posibilidad para generar iniciativas que se proyecten homogéneamente, es decir, tomando en cuenta todas las aristas que influyen. La idea sería encaminar a Valdivia en virtud de un polo cultural que maneje a cabalidad tanto los aspectos económicos como políticos, de igual modo los humanos, culturales y urbanísticos. El gestor cultural es un profesional capacitado para idear propuestas que beneficien a la región aunando todos los criterios que deban tenerse en cuenta.

Es, sin duda, una oportunidad para la ciudad y para el país para responder con altura de miras y profesionalidad, y no quedarse en el mero evento cultural, el cual nace y se agota en sí mismo. La presión que ejerce que muchos países estén mirando a Valdivia como representante cultural de América para el 2016 debiera significar una nueva forma de pensar la producción de significados locales con el fin de mostrarlos al mundo. 


\section{Bibliografía}

ASPILLAGA FARIÑA, Alejandra (coord.) (2014): Mapeo de las Industrias Creativas en Chile: caracterización y dimensionamiento. Santiago: Gobierno de Chile, Consejo Nacional de la Cultura y las Artes.

BALIBREA, M. Paz (2011): "Barcelona: del modelo a la marca" [en línea], en Forum de cultura, democratizem la democracia. En: http://www.ebarcelona.org [Consulta: 22/05/2015].

BAYARDO, Rubens (2013): "Economía, Cultura y economía de la Cultura" [en línea]. Revista Voces en el Fénix, Año 4, núm. 29, pp. 14-21. En: http://www.youblisher.com/p/737367-Voces-en-el-Fenix-No-29-Elespiritu-de-la-colmena/ [Consulta: 10/03/2015].

GETINO, Octavio (2007): "La cultura como capital" [en línea], en octaviogetinocine.blogspot.com.

En: http://octaviogetinocine.blogspot.com.ar/2010/06/la-cultura-comocapital.html [Consulta: 27/05/2015]

GÓMEZ, Fernando (2004): "Arte, ciudadanía y espacio público" [en línea]. Revista Fundación César Manrique, núm. 5, marzo, pp. 36-51. En: http://www.ub.edu/escult/Water/N05/W05 3.pdf

[Consulta: 27/05/2015].

INTERNATIONAL BUREAU OF CULTURAL CAPITALS (2014): Declaratoria Ciudad Americana 2016 [en línea]. En: http://www.cacacc.org/docs/DV2016.pdf [Consulta: 10/03/2015].

LARSON, Gary (1997): American Canvas. Washington D.C.: National Endowment for the Arts.

PIEDRAS, Ernesto (2004): ¿Cuánto vale la cultura? Contribución económica de las industrias protegidas por el derecho de autor en México. México: Conaculta.

ROJAS, Camila (2010): Presente y pasado de una arquitectura regional: el casi de la ciudad de Valdivia. Santiago: Facultad de Arquitectura y Urbanismo.

TOLILA, Paul (2007): Economía da cultura. Sao Paulo: Editora Illuminuras.

YÚDICE, George (2012): El recurso de la cultura: Usos de la cultura en la era global. Barcelona: Gedisa. 